\title{
Calcul des courants de Foucault en trois dimensions par une formulation utilisant le potentiel vecteur magnétique et le potentiel scalaire électrique
}

\author{
G. Carron de la Morinais $\left({ }^{1}\right)$, G. Meunier $\left({ }^{1}\right)$ et C. Kieny $\left({ }^{2}\right)$ \\ (') Laboratoire d'Electrotechnique de Grenoble (LEG), CNRS-UA 355, ENSIEG, B.P. 46, 38402 Saint \\ Martin d'Hères, France \\ (2) Electricité de France, Direction des études et recherches, Service ERMEL, 1, Avenue du Général de \\ Gaulle, 92141 Clamart, France
}

(Reçu le 31 octobre 1989, révisé le 8 mars 1990, accepté le 23 avril 1990)

\begin{abstract}
Résumé. - Le calcul des courants induits en trois dimensions est primordial dans l'optique de la conception de matériel électrique. En effet, la plupart des dispositifs électriques fonctionnent en régime dynamique : transformateurs, alternateurs, machines asynchrones... Au sein de tous ces appareils apparaissent des phénomènes liés aux courants de Foucault. Une formulation permettant de les modéliser en régime harmonique est présentée ici. Elle utilise le potentiel vecteur magnétique $A$ et le potentiel scalaire électrique $V$. Les régions non conductrices, quant à elles, sont modélisées grâce au potentiel scalaire magnétique. Le couplage entre ces deux méthodes est ensuite développé. Enfin, un exemple est présenté.
\end{abstract}

\begin{abstract}
The ultimate goal of Computer Aided Design (CAD) codes is to simulate efficiently electric equipment. If there are nowadays accurate programs to model three dimensional static problems, the case of three dimensional time varying problems is not fully understood. This has important repercussions because, in practice, most electric machines function under dynamic constrains. A three dimensional eddy current formulation using the magnetic vector potential and the electric scalar potential is presented in this paper. Non conductive regions are modeled using magnetic scalar potential. Finally, an exemple will illustrate our approach.
\end{abstract}

\section{Equations à résoudre. Hypothèses.}

Le problème magnétodynamique que nous présentons ici consiste à résoudre les équations de Maxwell en régime linéaire harmonique : les matériaux sont linéaires, et toutes les grandeurs fonction du temps varient sinusoïdalement avec celui-ci.

champs étudiés est grande devant la dimension des géométries. Ainsi, on peut négliger les courants de déplacement par rapport aux courants induits. Dans la plupart des dispositifs électrotechniques, cette simplification est justifiée.

Les géométries à étudier sont en général composées des parties suivantes:

- des bobines inductrices dans lesquelles ne se développent pas de courants de Foucault ;

- des conducteurs où peuvent circuler des courants induits;
- une boîte, qui englobe le tout. Elle ne contient ni courants source ni courants induits, et est composée d'air et éventuellement de pièces magnétiques isolantes d'un point de vue électrique.

L'exemple suivant (Fig. 1), le plus simple qui soit, servira de support à cette étude. Une boîte d'air

courants source $J_{s}$, et un conducteur de perméabilité $\boldsymbol{\mu}$ et de conductivité $\boldsymbol{\sigma}$.

Les équations à résoudre sont alors, en régime quasi-stationnaire :

$$
\begin{aligned}
& \operatorname{div} E=0 \\
& \operatorname{div} B=0 \\
& \operatorname{rot} E=-\partial B / \partial t \\
& \operatorname{rot} H=J .
\end{aligned}
$$

Où $E$ est le champ électrique, $H$ le champ magnétique, $B$ l'induction magnétique, et $J$ la densité de courants. 


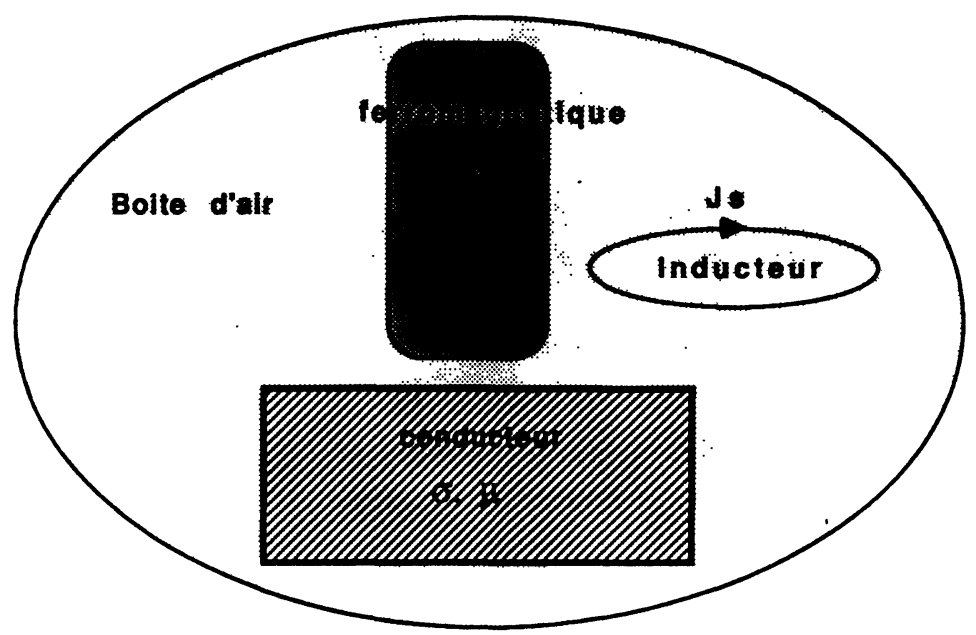

Fig. 1. - Exemple de géométrie.

[Example of geometry.]

On dispose de plus des relations constitutives des matériaux :

$$
\begin{aligned}
B & =\mu H \\
J & =\sigma E .
\end{aligned}
$$

Où $\mu$ est la perméabilité magnétique et $\sigma$ la conductivité électrique.

La relation (4) permet d'écrire :

$$
\operatorname{div} J=0 \text {. }
$$

Qui est l'équation de conservation de la densité de courants.

Les champs de vecteurs vérifient les conditions de continuité suivantes aux interfaces entre deux régions de propriétés différentes :

$$
\begin{aligned}
\{B \cdot N\} & =0 \\
\{J \cdot N\} & =0 \\
\{H \times N\} & =0 \\
\{E \times N\} & =0 .
\end{aligned}
$$

En notant $N$ la normale à l'interface, et $\{X\}$ la variation d'une variable $X$. Ces relations sont consécutives aux équations de Maxwell. Elles expriment les conditions que doivent remplir les champs de vecteurs. Les composantes normales du vecteur induction magnétique et du vecteur densité de courant sont toujours continues. Il en est de même pour les composantes tangentielles des vecteurs champ magnétique et champ électrique.

\section{Formulations.}

Les équations précédentes permettent théoriquement de décrire tous les systèmes quasi-stationnaires, donc la plupart des dispositifs utilisés en électrotechnique. Toutefois, une mise en forme préliminaire s'impose afin de réduire le nombre d'inconnues et le nombre d'équations à résoudre. Si en deux dimensions, le potentiel vecteur, qui n'a alors qu'une composante, fait l'unanimité, en trois dimensions, il n'y a pas de véritable consensus sur la meilleure formulation. De nombreuses méthodes ont éte proposées utilisant des variables de type potentiel ou de type champ. Malgré quelques variantes qui diffèrent par exemple sur les conditions de jauge, on peut les décomposer en quatre grandes familles : les formulations $A-V, A^{*}$ ou $E, T-\Omega$, et $H[2,10,13]$.

Une formulation utilisant le potentiel vecteur magnétique $A$ et le potentiel scalaire électrique $V$ est présentée ici.

2.1 FORMULATION DANS LES RÉGIONS CONDUCTRICES. MÉthode $A-V$. Compte tenu de la relation (2), à savoir: $\operatorname{div} B=0, B$ dérive d'un potentiel vecteur $A$ tel que :

$$
B=\operatorname{rot} A
$$

et l'équation (3) implique l'existence d'un potentiel scalaire électrique $V$ tel que :

$$
E=-\partial A / \partial t-\operatorname{grad} V .
$$

Alors, il vient :

$$
J=\sigma(-\partial A / \partial t-\operatorname{grad} V) .
$$

En remplaçant les expressions de $B, E$, et $J$ dans les équations (3) et (4) et en les combinant, on obtient la relation :

$\operatorname{rot}[\nu(\operatorname{rot} A)]+\sigma(\partial A / \partial t+\operatorname{grad} V)=0$.

Où $\nu$ est l'inverse de la perméabilité magnétique $\mu: \nu=1 / \mu$.

Si les potentiels $A$ et $V$ sont continus, la continuité de la composante normale de l'induction magnétique 
et de la composante tangentielle du champ électrique sont immédiates.

Aux interfaces où la perméabilité passe de $\mu_{1}$ à $\mu_{2}$, il faut assurer la continuité de la composante tangentielle du champ magnétique, soit la condition, en notant $N$ un vecteur normal :

$$
\left[\nu_{1} \operatorname{rot} A_{1}\right] \times N=\left[\nu_{2} \operatorname{rot} A_{2}\right] \times N .
$$

La condition de conservation de la composante normale de la densité de courant $J$ impose quant à elle :

- aux interfaces où la conductivité subit une discontinuité :

$$
\sigma_{1} E_{1} \cdot N=\sigma_{2} E_{2} \cdot N
$$

soit, en termes de potentiels :

$$
\begin{aligned}
\sigma_{1}\left(-\partial A_{1} / \partial t-\right. & \left.\operatorname{grad} V_{1}\right) \cdot N= \\
& =\sigma_{2}\left(-\partial A_{2} / \partial t-\operatorname{grad} V_{2}\right) \cdot N
\end{aligned}
$$

- à l'interface entre une région conductrice et une région isolante :

$$
\sigma_{1} E_{1} \cdot N=0
$$

c'est-à-dire :

$$
\sigma_{1}\left(-\partial A_{1} / \partial t-\operatorname{grad} V_{1}\right) \cdot N=0
$$

L'équation (15), associée aux conditions d'interfaçage précédentes est vérifiée par une infinité de couples $(A, V)$. Le potentiel vecteur $A$ est défini au gradient d'une fonction scalaire $f$ près, et $V$ à la dérivée par rapport au temps de cette fonction $f:$ si $(A, V)$ est un couple solution, $\left(A^{\prime}, V^{\prime}\right)$, tel que

$$
\begin{aligned}
& A^{\prime}=A+\operatorname{grad}(f) \\
& V^{\prime}=V-\partial f / \partial t
\end{aligned}
$$

sera aussi solution. Il faut ici, pour avoir unicité de la solution en potentiel imposer une condition supplémentaire, une condition de jauge. Les deux jauges les plus utilisées sont les jauges de Coulomb $(\operatorname{div} A=0) \quad[1,8,11]$ et de Lorentz $(\operatorname{div} A=$ $-\sigma \mu V)$ [3].

Cette méthode est très lourde à utiliser. En effet, les équations à résoudre comportent une inconnue vectorielle - qui a trois composantes - et une permet de prendre en compte les discontinuités de conductivité, ce qui la rend tout de même intéressante.

2.2 FORMULATION DANS LES RÉGIONS NON CONDUCTRICES. MÉTHODE DU POTENTIEL RÉDUIT. - Dans les régions non conductrices, les équations de Maxwell se simplifient par le fait que la conductivité est nulle. Le système d'équations se réduit à :

$$
\begin{aligned}
& \operatorname{div} B=0 \\
& \operatorname{rot} H=J_{s} .
\end{aligned}
$$

Où $J_{\mathrm{s}}$ est la densité de courants source dans l'inducteur « Ind ».

Il est intéressant d'utiliser une formulation scalaire dans les régions non conductrices, on travaille ainsi avec trois fois moins d'inconnues que si l'on avait utilisé une formulation vectorielle. En revanche, le couplage avec les régions où est utilisé le potentiel vecteur est délicat, et des problèmes topologiques peuvent se poser dans les régions non simplement connexes.

La formulation utilisant le potentiel scalaire réduit a été choisie pour décrire les régions non conductrices $[4,7,12]$.

Le principe de cette méthode est de décomposer le champ $H$ en deux parties :

$$
H=H_{\mathrm{j}}+H_{\mathrm{r}} .
$$

Avec :

$H_{\mathrm{j}} \quad$ : champ qui serait créé par l'inducteur s'il était seul dans l'espace vide;

$H_{\mathrm{r}}$ : champ créé par la réaction des parties magnétiques ou conductrices, lorsqu'elles sont soumises au champ $H_{\mathrm{j}}$.

$H_{\mathrm{j}}$ au point $M$ est calculable par la formule de Biot et Savart :

$H_{\mathrm{j}}(M)=(1 / 4 \pi) \int_{\text {Ind }}\left[\left(J_{\mathrm{s}}(P) \times r\right) /(r)^{3}\right] \mathrm{d} P$

$P$ étant un point appartenant à l'inducteur, $r$ étant le vecteur $M P$.

$H_{\mathrm{j}}$ vérifie le théorème d'Ampère, tandis que $H_{\mathrm{r}}$ est à rotationnel nul :

$$
\operatorname{rot}\left(H_{\mathrm{j}}+H_{\mathrm{r}}\right)=J_{\mathrm{s}}
$$

avec :

$$
\begin{aligned}
& \operatorname{rot}\left(H_{\mathrm{j}}\right)=J_{\mathrm{s}} \\
& \operatorname{rot}\left(H_{\mathrm{r}}\right)=0 .
\end{aligned}
$$

D'après (25), on peut écrire que $H_{\mathrm{r}}$ dérive d'un gradient $\phi_{\mathrm{r}}$ :

$$
H_{\mathrm{r}}=-\operatorname{grad} \phi_{\mathrm{r}} .
$$

L'équation qu'on va résoudre est obtenue en rempla-

$$
\operatorname{div}\left[\mu\left(-\operatorname{grad} \phi_{\mathrm{r}}+H_{\mathrm{j}}\right)\right]=0 \text {. }
$$

Or :

$$
\operatorname{div}\left(\mu H_{\mathrm{j}}\right)=0
$$

dans les volumes où la perméabilité $\mu$ est constante. Soit, à résoudre :

$$
\begin{aligned}
\operatorname{div}\left[\mu\left(-\operatorname{grad} \phi_{\mathrm{r}}\right)\right] & =0 \\
H & =H_{\mathrm{j}}+H_{\mathrm{r}}
\end{aligned}
$$

dans les volumes où la perméabilité $\mu$ est constante. 
La conservation de la composante tangentielle du champ magnétique $H$ est vérifiée si le potentiel $\phi_{\mathrm{r}}$ utilisé est continu, mais il convient d'assurer la continuité de la composante normale du vecteur induction magnétique $B$ à une interface entre deux régions de perméabilités $\mu_{1}$ et $\mu_{2}$; cette condition s'écrit, en notant $N$ un vecteur normal à l'interface :

$\left[\mu_{1}\left(H_{\mathrm{j}}-\operatorname{grad} \phi_{\mathrm{r} 1}\right)\right] . N=\left[\mu_{2}\left(H_{\mathrm{j}}-\operatorname{grad} \phi_{\mathrm{r} 2}\right)\right] . N$.

Cette méthode, très séduisante sur le plan théorique, implique des problèmes numériques lorsque les perméabilités des matériaux non conducteurs sont importantes. En effet, pour obtenir le champ total dans les régions perméables, il faut faire la somme de deux champs de modules très voisins, et de signes opposés. Ceci implique une très mauvaise précision. Un moyen d'éviter cette difficulté est de travailler en potentiel scalaire total dans les régions perméables, où il n'y a pas, en général, circulation de courants. Le potentiel total, quant à lui, fournit une bonne précision dans les régions perméables [Simkin, 78].

Un autre problème, et non des moindres, posé par l'utilisation d'un potentiel scalaire dans les régions non conductrices est de type topologique. Un exemple simple l'illustre bien. Imaginons un tore dans lequel circulent des courants induits, l'extérieur du tore étant décrit en potentiel scalaire. Alors, la circulation du champ suivant un contour fermé dans l'air n'est pas la même selon que l'on entoure une section du tore ou non. Le potentiel scalaire est alors multiforme. Pour que le potentiel scalaire soit uniforme, il faut introduire une coupure interdisant l'une des deux circulations [6, 14].

Remarquons pour terminer qu'il est nécessaire d'assurer les conditions de continuité du champ aux interfaces entre les régions de formulations différentes. C'est le problème du couplage entre formulations.

\section{Application à la méthode des éléments finis.}

Etant donné qu'aucune des équations introduites dans le paragraphe précédent ne peut être résolue d'une manière générale par des méthodes analytiques, une méthode d'approximation doit leur être appliquée. La méthode des éléments finis a été choisie, de part la très grande facilité à l'appliquer sur toutes sortes de géométries.

Dans l'approximation des éléments finis, le domaine à étudier est décomposé en petits éléments, et une fonction approchant la solution, définie par une interpolation sur chaque élément, est ensuite recherchée. Les éléments de Lagrange, qui assurent la continuité de la variable inconnue sur le domaine, sont utilisés.

L'interpolation dans un élément, polynomiale, est effectuée en fonction des valeurs de la fonction inconnue aux nœuds de cet élément.

La fonction approchée de la solution vérifie les équations aux dérivées partielles à résoudre d'une manière faible, au sens des distributions; les conditions de continuité des champs seront donc elles aussi vérifiées d'une manière faible.

Les équations aux dérivées partielles à résoudre sont ainsi transformées en un système fini d'équations algébriques qu'il convient de résoudre pour obtenir la solution du problème posé.

Pour discrétiser les équations aux dérivées partielles, la méthode projective de Galerkine, ou encore méthode des résidus pondérés, est utilisée.

3.1 Formulation $A-V$. $-\mathrm{La}$ formulation $A-V$ est ici développée dans le cadre de la méthode des éléments finis.

La jauge de Coulomb, qui a été choisie, s'écrit :

$$
\operatorname{div}(A)=0 .
$$

L'équation $\operatorname{div} J=0$, équivalente à la suivante :

$$
\operatorname{div}[\sigma(\partial A / \partial t+\operatorname{grad} V)]=0
$$

peut alors s'écrire (dans les régions où $\sigma$ est cons$\operatorname{tant)}$ :

$$
\operatorname{div}[\sigma(\operatorname{grad} V)]=0 .
$$

Ainsi, cette équation n'est plus une conséquence de l'équation (15). Il est donc nécessaire de résoudre les équations suivantes :

$$
\begin{aligned}
\operatorname{rot}[\nu(\operatorname{rot} A)]+\sigma(\partial A / \partial t+\operatorname{grad} V) & =0 \\
\operatorname{div}[\sigma(\operatorname{grad} V)] & =0 .
\end{aligned}
$$

Dans l'hypothèse du régime harmonique de pulsation $\omega$, on peut utiliser la représentation complexe. En notant abusivement de la même manière une variable fonction du temps et sa représentation complexe, seul l'opérateur $[\partial / \partial t]$ - transformé en $[j \omega]$ - est modifié dans les équations. (34) et (35) deviennent alors :

$$
\begin{aligned}
\operatorname{rot}[\nu(\operatorname{rot} A)]+\sigma(j \omega A+\operatorname{grad} V) & =0 \\
\operatorname{div}[\sigma(\operatorname{grad} V)] & =0 .
\end{aligned}
$$

Et les conditions de continuité (17) et (18), s'écrivent :

$$
\begin{gathered}
\sigma_{1}\left(-j \omega A_{1}-\operatorname{grad} V_{1}\right) \cdot N= \\
=\sigma_{2}\left(-j \omega A_{2}-\operatorname{grad} V_{2}\right) \cdot N \\
\sigma_{1}\left(-j \omega A_{1}-\operatorname{grad} V_{1}\right) \cdot N=0 .
\end{gathered}
$$

La méthode de Galerkine est appliquée aux équations (36) et (37) en intégrant respectivement (36) multipliée par une fonction de pondération $W$, et (37) multipliée par une fonction de pondération 
$w$. Dans une région conductrice $\Omega$, de frontière $\Gamma$, on obtient les équations suivantes :

$\int_{\Omega} W[\operatorname{rot}(\nu \operatorname{rot} A)+\sigma(j \omega A+\operatorname{grad} V)] \mathrm{d} \Omega=0$

$$
\int_{\Omega} w[\operatorname{div} \sigma(\operatorname{grad} V)] \mathrm{d} \Omega=0 .
$$

Le théorème de Green permet de réduire l'ordre de ces équations :

$\int_{\Omega}[(\operatorname{rot} W)(\nu \operatorname{rot} A)+W \sigma(j \omega A+\operatorname{grad} V)] \mathrm{d} \Omega$

$$
-\int_{\Gamma} W(\nu)[(\operatorname{rot} A) \times N] \mathrm{d} \Gamma=0
$$

$\int_{\Omega}(\operatorname{grad} w) \sigma \operatorname{grad} V \mathrm{~d} \Omega-\int_{\Gamma} w \sigma(\operatorname{grad} V) \cdot N \mathrm{~d} \Gamma$

$$
+\int_{\Gamma_{\mathrm{i}}} w\{\sigma(\operatorname{grad} V)\} \cdot N \mathrm{~d} \Gamma=0 .
$$

$\Gamma_{\mathrm{i}}$ est l'ensemble des surfaces sur lesquelles la conductivité est discontinue, induisant une discontinuité de $[\sigma \operatorname{grad} V$ ], et $N$ est la normale sortant du volume $\Omega$ ou la normale à la surface $\Gamma_{\mathrm{i}}$. On note $\{X\}$ une variation de la variable $X$.

Or, selon (12) et (5) :

$$
\int_{\Gamma} W(\nu)[(\operatorname{rot} A) \times N] \mathrm{d} \Gamma=\int_{\Gamma} W(H \times N) \mathrm{d} \Gamma .
$$

D'après (39), à l'interface entre une région conductrice et une région isolante, il vient :

$$
\int_{\Gamma} w \sigma(\operatorname{grad} V) \cdot N \mathrm{~d} \Gamma=-\int_{\Gamma} w \sigma(j \omega A) \cdot N \mathrm{~d} \Gamma .
$$

D'après (38), au niveau des interfaces $\Gamma_{\mathrm{i}}$, où la conductivité est discontinue, le gradient de $V$ est discontinu. Il faudra vérifier :

$$
\begin{aligned}
\int_{\Gamma_{\mathrm{i}}} w\{\sigma(\operatorname{grad} V)\} & N \mathrm{~d} \Gamma= \\
& =-\int_{\Gamma_{\mathrm{i}}} w\{\sigma(j \omega A)\} \cdot N \mathrm{~d} \Gamma .
\end{aligned}
$$

En notant toujours \{\} une variation. On peut le terme en $A$.
Les équations (40) et (41) s'écrivent alors de la manière suivante :

$$
\begin{aligned}
\int_{\Omega}[(\operatorname{rot} W) \nu \operatorname{rot} A & +W \sigma(j \omega A+\operatorname{grad} V)] \mathrm{d} \Omega- \\
& -\int_{\Gamma} W(H \times N) \mathrm{d} \Gamma=0
\end{aligned}
$$

$\int_{\Omega}(\operatorname{grad} w) \sigma \operatorname{grad} V \mathrm{~d} \Omega+\int_{\Gamma_{\mathrm{i}}} w\{\sigma j \omega A\} \cdot N \mathrm{~d} \Gamma$

$$
+\int_{\Gamma} w(\sigma j \omega A) \cdot N \mathrm{~d} \Gamma=0 .
$$

La géométrie étudiée est découpée en éléments finis. Les potentiels sont alors interpolés en fonction de leurs valeurs aux nœuds du maillage de la manière suivante :

$$
\begin{aligned}
& V=\sum_{i=1}^{N_{\mathrm{n}}} \alpha_{i} V_{i} \\
& A=\sum_{i=1}^{N_{\mathrm{n}}} \alpha_{i}\left[I d_{3}\right] A_{i} .
\end{aligned}
$$

Avec :

$N_{\text {n }} \quad$ : Nombre total de nœuds du domaine.

$\alpha_{i} \quad$ : Fonction de forme associée au nœud $i$.

$\left[I d_{3}\right]$ : Matrice identité $3 \times 3$.

$V_{i} \quad$ : Valeur de $V$ au nœud $i$.

$A_{i} \quad$ : Valeur de $A$ au nœud $i$.

Les $N_{\mathrm{n}}$ fonctions de forme associées aux $N_{\mathrm{n}}$ nœuds du maillage sont choisies comme fonctions de pondération. $W_{j}$ et $w_{j}$ s'écrivent alors :

$$
\begin{aligned}
w_{j} & \equiv \alpha_{j} \\
W_{j} & \equiv \alpha_{j}\left[I d_{3}\right] .
\end{aligned}
$$

Les variables s'écrivent en fonction des $W_{i}$ et des $w_{i}:$

$$
\begin{aligned}
& V=\sum_{i=1}^{N_{\mathrm{n}}} w_{i} V_{i} \\
& A=\sum_{i=1}^{N_{\mathrm{n}}} W_{i} A_{i} .
\end{aligned}
$$

En remplaçant les fonctions de pondération par les fonctions de forme, et en écrivant les potentiels s 42 et 43 deviennent, pour chacune des $N_{\mathrm{n}}$ fonctions :

$$
\begin{array}{r}
\int_{\Omega}\left[\left(\operatorname{rot} W_{i}\right) \nu \sum_{j=1}^{N_{\mathrm{n}}}\left(\operatorname{rot} W_{j}\right) A_{j}+W_{i} \sigma\left(j \omega \sum_{j=1}^{N_{\mathrm{n}}} W_{j} A_{j}+\sum_{j=1}^{N_{\mathrm{n}}}\left(\operatorname{grad} w_{j}\right) V_{j}\right)\right] \mathrm{d} \Omega-\int_{\Gamma} W_{i}(H \times N) \mathrm{d} \Gamma=0 \\
\int_{\Omega}\left(\operatorname{grad} w_{i}\right) \sigma \sum_{j=1}^{N_{\mathrm{n}}}\left(\operatorname{grad} w_{j}\right) V_{j} \mathrm{~d} \Omega+\int_{\Gamma_{\mathrm{i}}} w_{i} j \omega\{\sigma\}\left(\sum_{j=1}^{N_{\mathrm{n}}} W_{j} A_{j}\right) \cdot N \mathrm{~d} \Gamma+ \\
+\int_{\Gamma} w_{i} j \omega \sigma\left(\sum_{j=1}^{N_{\mathrm{n}}} W_{j} A_{j}\right) \cdot N \mathrm{~d} \Gamma=0 .
\end{array}
$$


Soit, en permutant les symboles de sommation :

$$
\begin{aligned}
& \sum_{j=1}^{N_{\mathrm{n}}}\left[\int_{\Omega}\left(\left(\operatorname{rot} W_{i}\right) \nu\left(\operatorname{rot} W_{j}\right)+W_{i}\left(j \omega \sigma W_{j}\right)\right) \mathrm{d} \Omega\right]\left[A_{j}\right]+ \\
& +\sum_{j=1}^{N_{\mathrm{n}}}\left[\int_{\Omega}\left(W_{i}\left(\sigma \operatorname{grad} w_{j}\right)\right) \mathrm{d} \Omega\right]\left[V_{j}\right]-\left[\int_{\Gamma} W_{i}(H \times N) \mathrm{d} \Gamma\right]=0 \\
& \sum_{j=1}^{N_{\mathrm{n}}}\left[\int_{\Omega}\left(\operatorname{grad} w_{i}\right) \sigma\left(\operatorname{grad} w_{j}\right) \mathrm{d} \Omega\right]\left[V_{j}\right]+\sum_{j=1}^{N_{\mathrm{n}}}\left[\int_{\Gamma_{\mathrm{i}}} j \omega\{\sigma\} w_{i} W_{j} \cdot N \mathrm{~d} \Gamma\right]\left[A_{j}\right]+ \\
& +\sum_{j=1}^{N_{\mathrm{n}}}\left[\int_{\Gamma} j \omega \sigma w_{i} W_{j} \cdot N \mathrm{~d} \Gamma\right]\left[A_{j}\right]=0 \text {. }
\end{aligned}
$$

Ceci forme un système linéaire de $4 N_{\text {n }}$ équations à $4 N_{\mathrm{n}}$ inconnues. Pour tout $i$ variant de 1 à $N_{\mathrm{n}}$ :

$$
\begin{aligned}
& \sum_{j=1}^{N_{\mathrm{n}}} R_{i j} A_{j}+\sum_{j=1}^{N_{\mathrm{n}}} S_{i j} V_{j}=P_{i} \\
& \sum_{j=1}^{N_{\mathrm{n}}} T_{i j} A_{j}+\sum_{j=1}^{N_{\mathrm{n}}} U_{i j} V_{j}=0 .
\end{aligned}
$$

Seuls les termes dont les indices $i$ et $j$ correspondent à deux nœuds d'un même élément sont non nuls. Il est à noter que ce système n'est pas symétrique.

Les coefficients de la matrice s'écrivent:

$$
\begin{aligned}
& R_{i j}= {\left[\int_{\Omega}\left(\left(\operatorname{rot} W_{i}\right) \nu\left(\operatorname{rot} W_{j}\right)+W_{i}\left(j \omega \sigma W_{j}\right)\right) \mathrm{d} \Omega\right] } \\
& S_{i j}= {\left[\int_{\Omega}\left(W_{i}\left(\sigma \operatorname{grad} w_{j}\right)\right) \mathrm{d} \Omega\right] } \\
& T_{i j}= {\left[\int_{\Gamma_{\mathrm{i}}} j \omega\{\sigma\} w_{i} W_{j} \cdot N \mathrm{~d} \Gamma+\right.} \\
&\left.+\int_{\Gamma} j \omega \sigma w_{i} W_{j} \cdot N \mathrm{~d} \Gamma\right]
\end{aligned}
$$$$
U_{i j}=\left[\int_{\Omega}\left(\operatorname{grad} w_{i}\right) \sigma\left(\operatorname{grad} w_{j}\right) \mathrm{d} \Omega\right]
$$$$
P_{i}=\left[\int_{\Gamma} W_{i}(H \times N) \mathrm{d} \Gamma\right] \text {. }
$$

Remarque. - Des conditions aux limites naturelles de type champ normal nul ou champ tangentiel nul font disparaître le terme $P_{i}$. Le terme $P_{i}$ n'apparaît que lors de couplages entre formulations, où il sert à assurer les conditions de passage du champ magnétique $H$.

3.2 MÉThode DU POTENTIEL RÉdUIT. - Il faut résoudre, dans chaque sous-région de perméabilité constante :

$$
\begin{aligned}
\operatorname{div}\left[\mu\left(-\operatorname{grad} \phi_{\mathrm{r}}\right)\right] & =0 \\
H & =H_{\mathrm{j}}+H_{\mathrm{r}} .
\end{aligned}
$$

Et l'on doit assurer la conservation de la composante normale du vecteur induction magnétique aux interfaces où la perméabilité magnétique subit une discontinuité.

De même que dans les parties précédentes, on intègre le produit de l'équation (44) par une fonction de pondération scalaire $w$ sur le domaine. Dans une région non conductrice $\Omega$, de frontière $\Gamma$, on obtient :

$$
\int_{\Omega} w \operatorname{div}\left[\mu\left(-\operatorname{grad} \phi_{\mathrm{r}}\right)\right] \mathrm{d} \Omega=0 .
$$

Le théorème de Green permet de réduire l'ordre de cette équation :

$$
\begin{array}{r}
\int_{\Omega}(\operatorname{grad} w) \mu \operatorname{grad} \phi_{\mathrm{r}} \mathrm{d} \Omega- \\
-\int_{\Gamma} w \mu\left(\operatorname{grad} \phi_{\mathrm{r}}\right) \cdot N \mathrm{~d} \Gamma \\
-\int_{\Gamma_{\mathrm{i}}} w\left\{\mu\left(\operatorname{grad} \phi_{\mathrm{r}}\right)\right\} \cdot N \mathrm{~d} \Gamma=0
\end{array}
$$

En notant toujours \{\} une variation. Les $\Gamma_{\mathrm{i}}$ sont les surfaces sur lesquelles la perméabilité subit une discontinuité, induisant une discontinuité de $\mu \operatorname{grad} \phi_{\mathrm{r}} . N$. Le terme intégral correspondant traduit le fait qu'à cette interface, la divergence de $\left[\mu\left(-\operatorname{grad} \phi_{\mathrm{r}}\right)\right]$ n'est pas nulle.

La condition de continuité de la composante normale de l'induction impose que la variation de $\left[-\mu\left(\operatorname{grad} \phi_{\mathrm{r}}\right)\right] . N$ soit l'opposée de celle de $\mu\left(H_{\mathrm{j}}\right) . N$, ce qui s'écrit :

$\int_{\Gamma_{\mathrm{i}}} w\left\{\mu\left(\operatorname{grad} \phi_{\mathrm{r}}\right)\right\} \cdot N \mathrm{~d} \Gamma=\int_{\Gamma_{\mathrm{i}}} w\left\{\mu H_{\mathrm{j}}\right\} \cdot N \mathrm{~d} \Gamma$.

On peut donc, aux interfaces $\Gamma_{\mathrm{i}}$, remplacer le terme surfacique fonction de $\operatorname{grad} \phi_{\mathrm{r}}$ par un terme fonction de $H_{\mathrm{j}}$. De même, selon (45), on peut écrire :

$\int_{\Gamma} w \mu\left(\operatorname{grad} \phi_{\mathrm{r}}\right) \cdot N \mathrm{~d} \Gamma=\int_{\Gamma} w \mu\left(H_{\mathrm{j}}-H\right) \cdot N \mathrm{~d} \Gamma$. 
Selon (5), on peut remplacer le terme fonction de $\mu H$ par un terme fonction de $B$ :

$$
\begin{aligned}
\int_{\Gamma} w \mu\left(\operatorname{grad} \phi_{\mathrm{r}}\right) \cdot N \mathrm{~d} \Gamma= \\
=\int_{\Gamma} w \mu\left(H_{\mathrm{j}}\right) \cdot N \mathrm{~d} \Gamma-\int_{\Gamma} w B \cdot N \mathrm{~d} \Gamma .
\end{aligned}
$$

L'équation (46) devient alors :

$$
\begin{gathered}
\int_{\Omega}(\operatorname{grad} w) \mu \operatorname{grad} \phi_{\mathrm{r}} \mathrm{d} \Omega+\int_{\Gamma} w(B) \cdot N \mathrm{~d} \Gamma- \\
-\int_{\Gamma_{\mathrm{i}}} w\left\{\mu H_{\mathrm{j}}\right\} \cdot N \mathrm{~d} \Gamma \\
-\int_{\Gamma} w\left(\mu H_{\mathrm{j}}\right) \cdot N \mathrm{~d} \Gamma=0 .
\end{gathered}
$$

L'intégrale surfacique fonction de $B$ a une contribution nulle sur les frontières où le champ normal est nul et sur les frontières où le champ tangentiel est nul. Cependant, aux interfaces entre régions conductrices et régions non conductrices, c'est elle qui va servir à coupler les formulations utilisées.

Toujours de la même manière, les régions non conductrices sont découpées en éléments finis. Et le potentiel est alors interpolé en fonction de ses valeurs aux nœuds du maillage :

$$
\phi_{\mathrm{r}}=\sum_{i=1}^{N_{\mathrm{n}}} \alpha_{i} \phi_{r i} .
$$

Avec :

$N_{\mathrm{n}}$ : Nombre total de nœuds du domaine.

$\alpha_{i}$ : Fonction de forme associée au nœud $i$.

$\phi_{\mathrm{r} i}$ : Valeur de $\phi_{\mathrm{r}}$ au nœud $i$.

Les $N_{\mathrm{n}}$ fonctions de forme associées aux $N_{\mathrm{n}}$ nœuds du maillage sont choisies comme fonctions de pondération. $w_{j}$ s'écrit alors :

$$
w_{j} \equiv \alpha_{j}
$$

Les variables s'écrivent en fonction des $w_{\mathrm{i}}$ :

$$
\phi_{\mathrm{r}}=\sum_{i=1}^{N_{\mathrm{n}}} w_{i} \phi_{\mathrm{r} i} \text {. }
$$

les fonctions de forme, et en écrivant les potentiels sous leur forme interpolée, l'équation (47) devient, pour chacune des $N_{\mathrm{n}}$ fonctions :

$$
\begin{aligned}
\int_{\Omega}\left(\operatorname{grad} w_{i}\right) & \mu \sum_{j=1}^{N_{\mathrm{n}}}\left(\operatorname{grad} w_{j}\right) \phi_{\mathrm{r} j} \mathrm{~d} \Omega+ \\
& +\int_{\Gamma} w_{i} B \cdot N \mathrm{~d} \Gamma=\int_{\Gamma} w_{i} \mu\left(H_{\mathrm{j}}\right) \cdot N \mathrm{~d} \Gamma \\
& +\int_{\Gamma_{\mathrm{i}}} w_{i}\left\{\mu\left(H_{\mathrm{j}}\right)\right\} \cdot N \mathrm{~d} \Gamma
\end{aligned}
$$

Soit, en permutant les symboles de sommation :

$$
\begin{gathered}
\sum_{j=1}^{N_{\mathrm{n}}}\left[\int_{\Omega}\left(\operatorname{grad} w_{i}\right) \mu\left(\operatorname{grad} w_{j}\right) \mathrm{d} \Omega\right]\left[\phi_{\mathrm{r} j}\right]+ \\
\quad \int_{\Gamma} w_{i} B \cdot N \mathrm{~d} \Gamma=\int_{\Gamma} w_{i} \mu\left(H_{\mathrm{j}}\right) \cdot N \mathrm{~d} \Gamma \\
+\int_{\Gamma_{\mathrm{i}}} w_{i}\left\{\mu\left(H_{\mathrm{j}}\right)\right\} \cdot N \mathrm{~d} \Gamma .
\end{gathered}
$$

Ces équations forment un système linéaire de $N_{\mathrm{n}}$ équations à $N_{\mathrm{n}}$ inconnues. Pour tout $i$ variant de 1 à $N_{\mathrm{n}}$ :

$$
\sum_{j=1}^{N_{\mathrm{n}}} L_{i j} \phi_{\mathrm{r} j}=Q_{i}+M_{i}
$$

Seuls les termes correspondant des indices $i$ et $j$ caractérisant deux nœuds d'un même élément sont non nuls.

Les coefficients de la matrice s'écrivent :

$$
\begin{aligned}
L_{i j}= & {\left[\int_{\Omega}\left(\operatorname{grad} w_{i}\right) \mu\left(\operatorname{grad} w_{j}\right) \mathrm{d} \Omega\right] } \\
Q_{i}= & {\left[\int_{\Gamma} w_{i} \mu\left(H_{\mathrm{j}}\right) \cdot N \mathrm{~d} \Gamma-\right.} \\
& \left.-\int_{\Gamma} w_{i} B \cdot N \mathrm{~d} \Gamma\right] \\
M_{i}= & \int_{\Gamma_{\mathrm{i}}} w_{i}\left\{\mu\left(H_{\mathrm{j}}\right)\right\} \cdot N \mathrm{~d} \Gamma .
\end{aligned}
$$

Remarque. - Le terme fonction de $B$ dans le second membre $P_{i}$ ne disparaît qu'aux limites, si l'on affecte à ces dernières des conditions naturelles de type champ normal nul ou champ tangentiel nul.

3.3 Couplage ENTRE FORMULATIONS. - Ici est développé le couplage servant à assurer les conditions de continuité entre les régions qu'on a modélisées par différentes formulations [5, 9].

Le couplage entre la formulation $A-V$ et la formulation utilisant le potentiel vecteur dans les régions non conductrices n'est pas présenté, ce couplage étant naturel. En effet, la continuité de la composante normale du vecteur induction magnétique est nature ement assurée par les éléments utilisés, les éléments de Lagrange. Et les contributions surfaciques fonctions de $[H \times N]$, égales en module et opposées en signe, se simplifient. La continuité de la composante tangentielle du champ magnétique est ainsi assurée de façon faible lors de la résolution du système linéaire.

A chaque nœud de la frontière $\Gamma$ entre région conductrice et région non conductrice, l'application de la méthode de Galerkine nous a fourni les équations suivantes, les deux premières coté conducteur, la dernière coté non conducteur : 


$$
\begin{aligned}
\sum_{j=1}^{N_{\mathrm{nc}}} R_{i j} A_{j}+\sum_{j=1}^{N_{\mathrm{nc}}} S_{i j} V_{j} & =P_{i} \\
\sum_{j=1}^{N_{\mathrm{nc}}} T_{i j} A_{j}+\sum_{j=1}^{N_{\mathrm{nc}}} U_{i j} V_{j} & =0 \\
\sum_{j=1}^{N_{\mathrm{ne}}} L_{i j} \phi_{\mathrm{r} j} & =Q_{i} .
\end{aligned}
$$

Avec : $N_{\text {nc }}, N_{\text {ne }}:$ Nombres de nœuds respectivement de la région conductrice (frontière comprise) et de la région non conductrice (frontière comprise).

Les seuls coefficients de la matrice qui interviennent sur la frontière, et qui sont définis par les conditions aux limites, sont $P_{i}$ et $Q_{i}$ :

$$
\begin{aligned}
P_{i} & =\left[\int_{\Gamma} W_{i}\left(H \times N_{\mathrm{c}}\right) \mathrm{d} \Gamma\right] \\
Q_{i} & =\left[\int_{\Gamma} w_{i}\left(\mu\left(H_{\mathrm{j}}\right) \cdot N_{\mathrm{e}}\right) \mathrm{d} \Gamma-\int_{\Gamma} w_{i} B \cdot\left(N_{\mathrm{e}}\right) \mathrm{d} \Gamma\right]
\end{aligned}
$$

$N_{\mathrm{c}}$ (respectivement $N_{\mathrm{e}}$ ) est la normale sortant de la région conductrice (respectivement non conductrice).

La démarche consiste à exprimer $P_{i}$ en fonction du potentiel $\phi_{\mathrm{r}}$, et $Q_{i}$ en fonction du potentiel $A$. $Q_{i}$ et $P_{i}$ ne dépendent pas de $V$, ce couplage est seulement magnétique, pas électrique. $Q_{i}$ et $P_{i}$ peuvent s'écrire :

$$
\begin{aligned}
& \int_{\Gamma} W_{i}\left(H \times N_{\mathrm{c}}\right) \mathrm{d} \Gamma= \\
& \quad=\int_{\Gamma} W_{i}\left[\left(H_{\mathrm{j}}-\operatorname{grad} \phi_{\mathrm{r}}\right) \times N_{\mathrm{c}}\right] \mathrm{d} \Gamma \\
& \int_{\Gamma} w_{i}(\mu(48) \\
& =\int_{\Gamma} w_{i}\left(\mu\left(H_{\mathrm{j}}\right)\right) \cdot N_{\mathrm{e}} \mathrm{d} \Gamma-\int_{\Gamma} w_{i} B \cdot N_{\mathrm{e}} \mathrm{d} \Gamma=
\end{aligned}
$$

Or, les potentiels s'écrivent sur la frontière, d'une manière interpolée :

$$
\begin{aligned}
& A=\sum_{i=1}^{N_{\mathrm{nf}}} W_{i} A_{i} \\
& \phi_{\mathrm{r}}=\sum_{i=1}^{N_{\mathrm{nf}}} w_{i} \phi_{\mathrm{r} i} .
\end{aligned}
$$

On les remplace par leur approximation, ce qui permet d'obtenir :

$$
\begin{aligned}
P_{i}= & \int_{\Gamma} W_{i}\left(-\operatorname{grad}\left(\sum_{i=1}^{N_{\mathrm{nf}}} w_{i} \phi_{\mathrm{r} i}\right) \times N_{\mathrm{c}}\right) \mathrm{d} \Gamma+ \\
& +\int_{\Gamma} W_{i}\left(H_{\mathrm{j}} \times N_{\mathrm{c}}\right) \mathrm{d} \Gamma
\end{aligned}
$$

$$
\begin{aligned}
Q_{i}= & \int_{\Gamma} w_{i}\left(\mu\left(H_{\mathrm{j}}\right)\right) \cdot N_{\mathrm{e}} \mathrm{d} \Gamma- \\
& -\int_{\Gamma} w_{i} \operatorname{rot}\left(\sum_{i=1}^{N_{\mathrm{nf}}} W_{i} A_{i}\right) \cdot N_{\mathrm{e}} \mathrm{d} \Gamma .
\end{aligned}
$$

Soit, en permutant les indices de sommation :

$$
\begin{aligned}
P_{i}= & \sum_{i=1}^{N_{\mathrm{n}}}\left[\int_{\Gamma} W_{i}\left(-\operatorname{grad}\left(w_{i}\right) \times N_{\mathrm{c}}\right) \mathrm{d} \Gamma\right]\left[\phi_{\mathrm{r} i}\right]+ \\
& +\int_{\Gamma} W_{i}\left(H_{\mathrm{j}} \times N_{\mathrm{c}}\right) \mathrm{d} \Gamma \\
Q_{i}= & \int_{\Gamma} w_{i}\left(\mu\left(H_{\mathrm{j}}\right)\right) \cdot N_{\mathrm{e}} \mathrm{d} \Gamma- \\
& -\sum_{i=1}^{N_{\mathrm{n}}}\left[\int_{\Gamma} w_{i} \operatorname{rot}\left(W_{i}\right) \cdot N_{\mathrm{e}} \mathrm{d} \Gamma\right]\left[A_{i}\right] .
\end{aligned}
$$

D'où le système définitif, dont les lignes correspondant au nœud numéroté $i$ s'écrivent:

$$
\begin{aligned}
& \sum_{j=1}^{N_{\mathrm{nc}}} R_{i j} A_{j}+ \sum_{j=1}^{N_{\mathrm{nc}}} S_{i j} V_{j}+\sum_{j=1}^{N_{\mathrm{nf}}} M_{i j} \phi_{\mathrm{r} j}=D_{i} \\
& \sum_{j=1}^{N_{\mathrm{nc}}} T_{i j} A_{j}+\sum_{j=1}^{N_{\mathrm{nc}}} U_{i j} V_{j}=0 \\
& \sum_{j=1}^{N_{\mathrm{nf}}} N_{i j} A_{j}+\sum_{j=1}^{N_{\mathrm{ne}}} L_{i j} \phi_{\mathrm{r} j}=K_{i} .
\end{aligned}
$$

Avec : $N_{\mathrm{nc}}, N_{\mathrm{nf}}, N_{\mathrm{ne}}$ : Nombres de nœuds respectivement de la région conductrice (frontière comprise), de la frontière, et de la région non conductrice (frontière comprise)

$$
\begin{aligned}
R_{i j}= & {\left[\int_{\Omega}\left[\left(\operatorname{rot} W_{i}\right) \nu\left(\operatorname{rot} W_{j}\right)+W_{i}\left(j \omega \sigma W_{j}\right)\right] \mathrm{d} \Omega\right] } \\
S_{i j}= & {\left[\int_{\Omega}\left(W_{i}\left(\sigma \operatorname{grad} w_{j}\right)\right) \mathrm{d} \Omega\right] } \\
T_{i j}= & {\left[\int_{\Gamma_{\mathrm{i}}} j \omega\{\sigma\} w_{i} W_{j} \cdot N \mathrm{~d} \Gamma+\right.} \\
& \left.+\int_{\Gamma} j \omega \sigma w_{i} W_{j} \cdot N \mathrm{~d} \Gamma\right]
\end{aligned}
$$$$
U_{i j}=\left[\int_{\Omega}\left(\operatorname{grad} w_{i}\right) \sigma\left(\operatorname{grad} w_{j}\right) \mathrm{d} \Omega\right]
$$$$
L_{i j}=\left[\int_{\Omega}\left(\operatorname{grad} w_{i}\right) \mu\left(\operatorname{grad} w_{j}\right) \mathrm{d} \Omega\right]
$$$$
K_{i}=\left[\int_{\Gamma_{\mathrm{i}}} w_{i}\left\{\mu H_{\mathrm{j}}\right\} \cdot N_{\mathrm{e}} \mathrm{d} \Gamma+\int_{\Gamma} w_{i}\left(\mu H_{\mathrm{j}}\right) \cdot N_{\mathrm{e}} \mathrm{d} \Gamma\right]
$$$$
M_{i j}=\left[\int_{\Gamma} W_{i}\left(\operatorname{grad}\left(w_{i}\right) \times N_{\mathrm{c}}\right) \mathrm{d} \Gamma\right]
$$$$
N_{i j}=\left[\int_{\Gamma} w_{i} \operatorname{rot}\left(W_{i}\right) \cdot N_{\mathrm{e}} \mathrm{d} \Gamma\right]
$$$$
D_{i}=\left[\int_{\Gamma} W_{i}\left(H_{\mathrm{j}} \times N_{\mathrm{c}}\right) \mathrm{d} \Gamma\right] \text {. }
$$ 
Remarques. - Il faut définir en chaque nœud de la surface une inconnue $A$, une inconnue $V$, et une inconnue $\phi_{r}$.

- Le couplage utilisé ici est de type faible. Les conservations de $B . N$ et de $H \times N$, exprimées par les équations (48) et (49), ne sont vérifiées que globalement, sur un élément surfacique, au travers de l'égalité de deux intégrales surfaciques, et pas en tout point.

- Enfin, ce couplage pose des problèmes numériques lorsque la perméabilité des volumes décrits en potentiel vecteur est grande. Une explication plausible est la suivante: comme le champ est presque normal à l'interface entre une région très perméable et une région peu perméable, le potentiel réduit est alors presque constant sur cette surface. Le calcul de $H \times N$ est donc très imprécis, d'où peut-être les problèmes que nous avons rencontrés.

\section{Exemple d'application.}

Les formulations développées ci-dessus ont été implantées dans le logiciel FLUX3D développé au Laboratoire d'Electrotechnique de Grenoble.
Pour valider la méthode proposée, nous disposions du logiciel TRIFOU. Ce programme, développé à Electricité De France, fonctionne avec succès depuis plusieurs années [BOSSAVIT-83]. Il utilise une formulation en champ magnétique appliquée sur des éléments d'arêtes dans les régions conductrices. Les régions non conductrices, quant à elles, sont modélisées par la méthode des intégrales de frontières.

Comme un programme permet d'échanger les données entre FLUX3D et TRIFOU, il a été possible d'utiliser le même maillage tétraédrique d'ordre un lors des résolutions, et d'exploiter les résultats fournis par les deux logiciels grâce au postprocesseur de FLUX3D [15].

Le problème que nous présentons a été proposé par Ren au Workshop qui s'est tenu à Bièvre en 1989 [10]. Il s'agit de calculer la répartition des courants de Foucault dans un cylindre creux, composé de deux matériaux de conductivités différentes (Fig. 2). Un courant sinusoïdal $(100 \mathrm{~A}, 50 \mathrm{~Hz})$ circule dans la bobine d'excitation, qui entoure le cylindre. Un huitième de la géométrie, seulement, est modélisé, de par les symétries existantes.

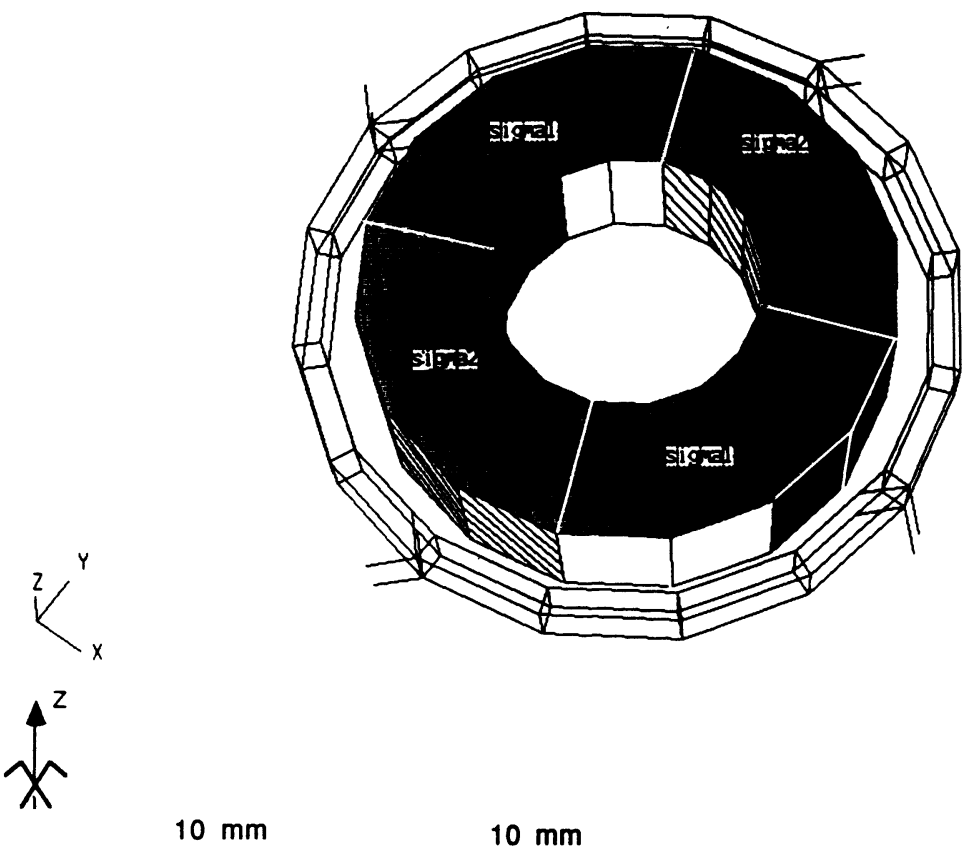

évidement conducteu $0 \mathrm{~mm} \quad 2 \mathrm{~mm}$

0

ind ur $r$

Fig. 2. - Géométrie étudiée. Vue d'ensemble et coupe suivant un plan radial.

[Studied geometry. Overview and cut along a radial plane.] 


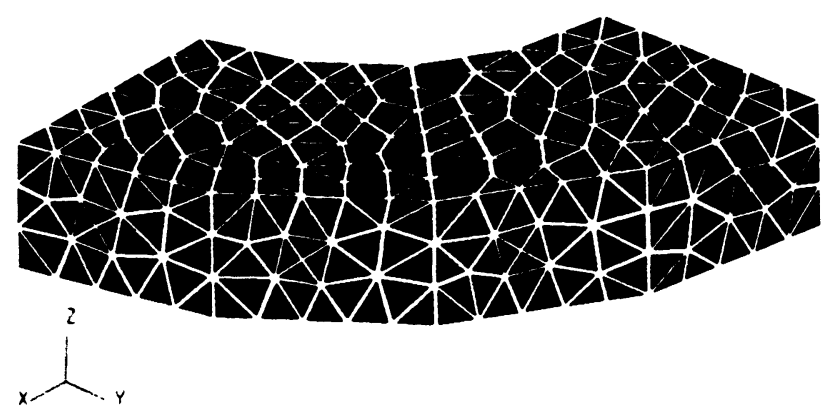

Fig. 3. - Maillage du cylindre creux.

[Hollow cylinder mesh.]

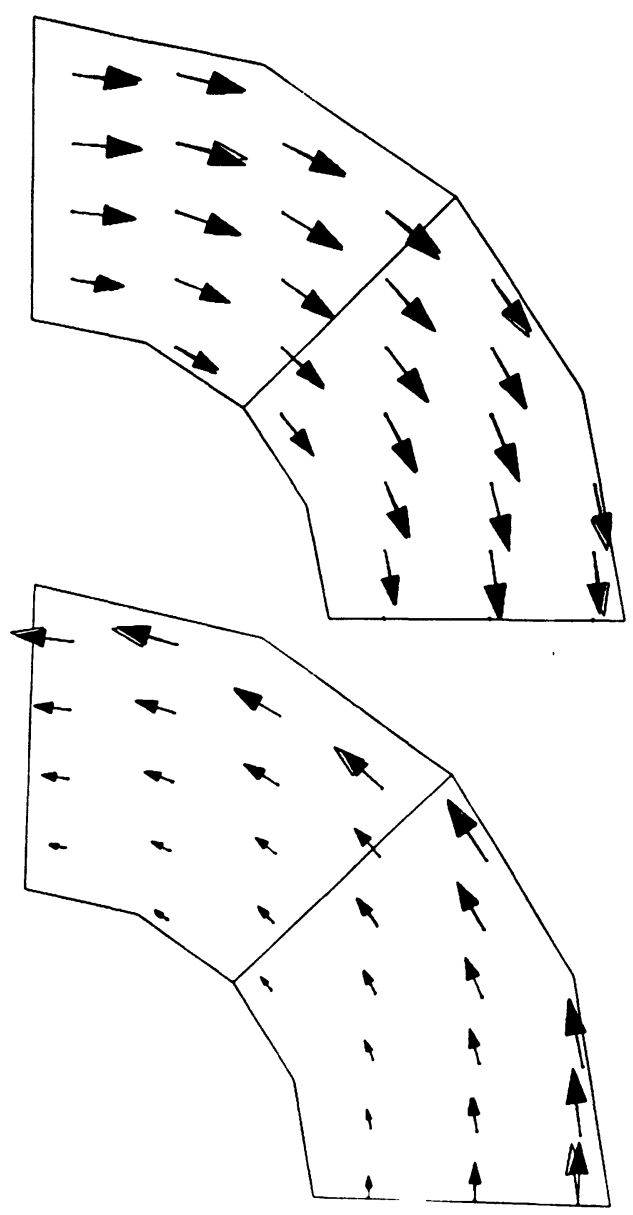

Fig. 4. - Densités de courant induits dans le plan $(z=0)$ réelles et imaginaires, dans le cas où la conductivité est constante: flèches blanches: TRIFOU, flèches noires : FLUX3D.

[Real and imaginary parts of the eddy current densities on the plane $(z=0)$, when conductivity is constant : white arrows : TRIFOU, black arrows : FLUX3D.]

Dans FLUX3D, le cylindre et l'extérieur sont décomposés en éléments finis, l'infini est pris en compte par troncature : on considère qu'à partir d'une distance du cylindre suffisante, le champ est nul (ici, quatre fois plus que le plus grand rayon du

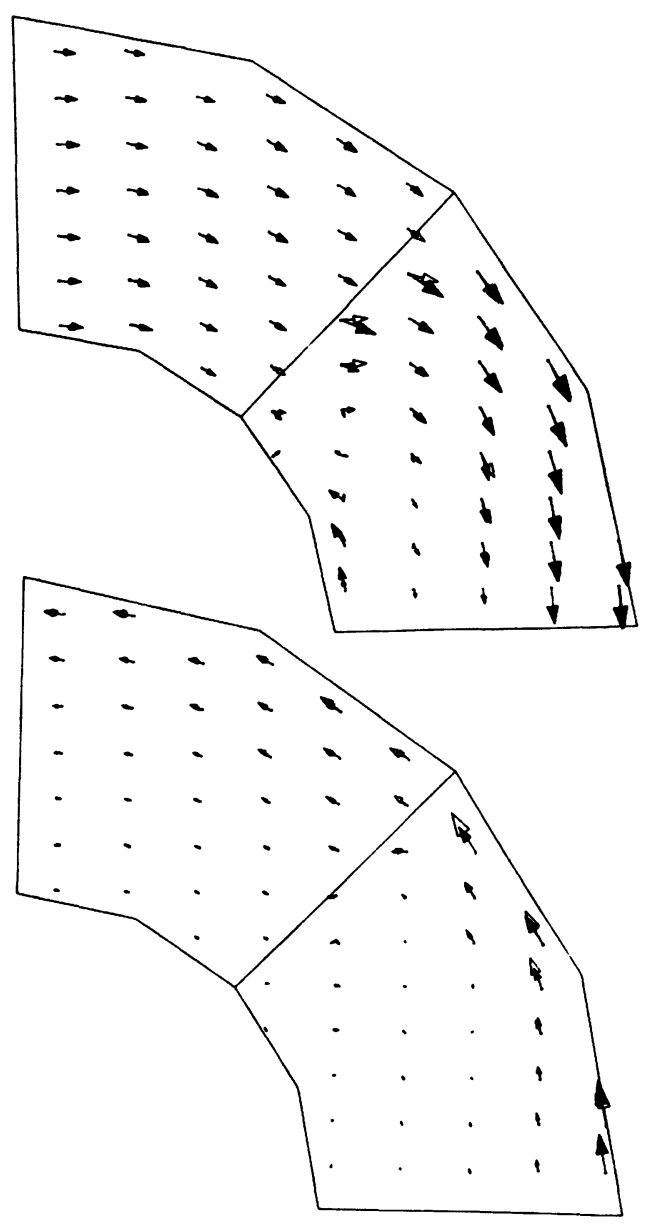

Fig. 5. - Densités de courant induits dans le plan $(z=0)$ réelles et imaginaires, quand il y a deux conductivités: flèches blanches: TRIFOU, flèches noires: FLUX3D.

[Real and imaginary parts of the eddy current densities on the plane $(z=0)$, when there are two conductivities: white arrows : TRIFOU, black arrows : FLUX3D.]

cylindre); c'est la limite du volume que l'on va mailler. Les parties conductrices sont décrites par les potentiels vecteur magnétique et scalaire électrique ; tandis que l'extérieur du cylindre est modélisé par le potentiel réduit.

Dans TRIFOU, seul le maillage du cylindre est conservé ; sur celui-ci, une formulation en champ $H$ est utilisée. L'extérieur est décrit par équations intégrales de frontière sur lesquelles est appliquée une formulation en potentiel réduit.

Le fait que le cylindre soit creux implique un problème de connexité. En effet, si l'on utilise le potentiel scalaire magnétique dans les régions non conductrices, celui-ci n'est alors pas défini de manière unique. Afin de surmonter cette difficulté, la couche d'air dans l'évidement du cylindre a été modélisé avec FLUX3D grâce à une formulation utilisant le potentiel vecteur (avec un terme de pénalité pour assurer l'unicité de $\boldsymbol{A}$ [4]). Avec 
TRIFOU le problème a été résolu en décrivant le trou comme un milieu conducteur de faible conductivité (bien que ce logiciel permette désormais de traiter le cas de conducteurs non simplement connexes [14]).

PREMIER CAS : CONDUCTIVITÉ UNIFORME SUR LE CYCLINDRE. - Ce cas, plus simple, permet de comparer les résultats obtenus par une formulation $A-V$ et des éléments nodaux, et ceux obtenus par une formulation en champ magnétique appliquée sur des éléments d'arête. La conductivité choisie est $10^{8}$ $(\Omega . \mathrm{m})^{-1}$. Les résultats comparés sont excellents (fig. 4), le maillage utilisé étant fin par rapport à la difficulté du problème.

DEUXIÈME CAS : DEUX CONDUCTIVITÉS. - On a choisi des conductivités de $10^{8}(\Omega . \mathrm{m})^{-1}$, et de
$5 \times 10^{8}(\Omega \cdot \mathrm{m})^{-1}$. Là encore, les résultats comparés obtenus par TRIFOU - et le champ magnétique ou par FLUX3D - et les potentiels - sont bons (Fig. 5). Un maillage plus fin permettrait sans doute de rapprocher encore les résultats l'un de l'autre.

\section{Conclusion.}

Dans cet article a été présentée une méthode de traitement de problèmes magnéto-dynamiques en trois dimensions en régime harmonique. Cette formulation a été implantée dans le logiciel FLUX3D et couplée à la formulation utilisant le potentiel réduit dans les régions non conductrices, afin de diminuer la taille de la matrice du système à résoudre. L'exemple proposé montre que la méthode proposée ici fournit des résultats tout à fait comparables à ceux obtenus par le logiciel TRIFOU.

\section{Bibliographie}

[1] BIRo O., PreIS K., On the use of the magnetic vector potential in the finite element analysis of three dimensional eddy currents, IEEE Trans. Magn. MAG-25 (1989) 92.

[2] Bossavit A., Verite J. C., The "Trifou " Code : Solving the 3-D Eddy-Currents Problem by Using $\mathrm{H}$ as State Variable, IEEE Trans. Magn. MAG-19 (1983) 2465.

[3] Bryant C. F., Emson C., Trowbridge C. W., A comparison of Lorentz gauge formulations in eddy current computations, COMPUMAG Tokyo 1989, à paraître dans IEEE Trans. Magn.

[4] Coulomb J. L., Analyse Tridimensionnelle des Champs Electriques et Magnétiques par la Méthode des Eléments Finis, Thèse d'Etat (juin 1981).

[5] Emson C. et Simkin J., An Optimal Method for 3D Eddy Currents, IEEE Trans. Magn. 19 (1983) 2450.

[6] Kotiuga P. R., On making cuts for magnetic scalar potentials in multiply connected domains, $J$. Appl. Phys. 61 (15 avril 1987).

[7] Mesquita R. C., Bastos J. P. A., Calculo de campos magnetostaticos nâo lineares tridimensium franco brésilien sur le calcul des champs électriques et magnétiques (1989) p. 58.

[8] Morisue T., A new formulation of the magnetic vector potential method in 3-D multiply connected regions, IEEE Trans. Magn. MAG-21 (1988) 110 .

[9] Pillsbury R. D. Jr, A three dimensional eddy
Current formulation using two potentials : the magnetic vector potential and total magnetic scalar potential, IEEE Trans. Mag. MAG-18 (1982) 492.

[10] Ren Z., Bouillault F., Razek A., Bossavit A. VERITE J.C., A New Hybrid Model Using Electric Field Formulation for 3-D Eddy current Problems, présenté à COMPUMAG Tokyo 1989, à publier dans IEEE Trans. Magn. (1990).

[11] Salon S. J., Peng J. P., Three Dimensional Eddy Currents Using a For Component Finite Element Formulation, IEEE Trans. Magn. MAG-20 (1984) 1994.

[12] Simkin J., Trowbridge C. W., Which potential ? A comparison of the various scalar and vector potentials for the numerical solution of nonlinear Poisson problems, Rapport du Rutherford Laboratory, ref. R.L.-78-009/B (janvier 1978).

[13] Trowbridge C. W., Introduction to eddy current formulations, Proc. du Eddy Current Workshop de Capri (1988).

[14] Verite J. C., Traitement du potentiel scalaire magnétique extérieur dans le cas d'un domaine multiplement connexe. Application au code TRI-

ches, série $C$ mathématiques informatique 1 (1986) 61.

[15] Chia B. T., Coulomb J. L., Sabonnadiere J. C., Verite J. C., Nicolas A., A Unified Database for Data Exchange in Electromagnetic Field Computation, présenté à COMPUMAG Tokyo 1989, à publier dans IEEE Trans. Magn. (1990). 